\title{
Serial laboratory reports and records for chemical analyses
}

\author{
L. G. WHITBY AND J. A. OWEN 1 \\ From the University Department of Clinical Chemistry, The Royal Infirmary, Edinburgh
}

SYNOPSIS A system of reporting laboratory data is described which provides serial and composite presentation of the results of chemical analyses as reports in the patients' notes in the form of copies of serial and composite records which are prepared and kept in the laboratory. The system has been used for seven months, providing reports for patients in 250 general medical beds, which generate an annual workload of approximately 90,000 chemical tests.

For several years, a combined request and report form (Fig. 1) has been used in conjunction with tests carried out in this laboratory. A detachable duplicate form of the 'no carbon required' type is retained in the laboratory for record purposes. These forms make small demands in terms of clerical work, but have various disadvantages:

1 Each report appears in the patient's notes on a separate piece of paper, which means that a comprehensive assessment of chemical results cannot readily be obtained.

2 Similarly, each duplicate report appears in the laboratory records as a single piece of paper, so that results of previous investigations cannot readily be inspected. Occasionally, a batch of forms is faulty and the duplicates are then valueless.

3 Because space is limited, several forms must be completed whenever a large number of tests is requested.

4 A significant percentage of forms becomes soiled and unfit for use as report forms.

5 Firm pressure is needed when filling out requests, as otherwise the duplicates may be too faint. If forms are in a pile when requests are being prepared, however, duplicates sometimes carry superimposed details of more than one request.

6 Each form costs $1.38 \mathrm{~d}$., but the above disadvantages all add to the cost per report.

An alternative method of reporting laboratory data has been developed which provides serial and composite presentation of results to the clinician, and serial and composite records in the laboratory. It is similar to the system described by Mason

1'Present address: The Alfred Hospital, Commercial Road, Prahran, S.I. Victoria, Australia.

Received for publication 27 May 1965.
(1962), but several practical points have been encountered which contribute to the successful operation of the system and were not covered in Mason's (1962) account. A full description of the reporting system is therefore presented.

In summary, the request form (Fig. 2) is used solely as a request form. For each new patient, a preprinted record card (Figs. 4 or 5, or both) is prepared in the laboratory and the results of analyses entered in the appropriate columns. A copy of the record card is made with a Rank-Xerox ${ }^{2} 914$ office copier and the copy issued as the report; the record card is retained in the laboratory. With subsequent requests, results are entered on the record card and a further copy, which now includes both previous and later results, is made and sent out as the report; this later cumulative report replaces the previous report in the patient's record folder.

\section{PREPARATION OF REPORTS AND LABORATORY RECORDS}

RECEPTION OF SPECIMENS, PREPARATION OF RECORD CARDS, MAIN RECORD FILE, AND CURRENT WORK FILE (FIGURE 3) Specimens arriving in the reception bay are allocated a letter and number; the letter changes each day and the numbers are issued in sequence as details for each patient are entered on the day-sheet. The identifying letter and number are also entered on the request form. Specimens are identified throughout the laboratory solely by their letter and number because of the large departmental workload $(300,000$ tests in 1964). The request forms are used for the preparation of record cards, and are then filed in order of registration in the request form file until results are ready to be reported. The record cards ${ }^{2}$ Rank-Xerox Ltd., 84-86 Great Portland Street, London. 


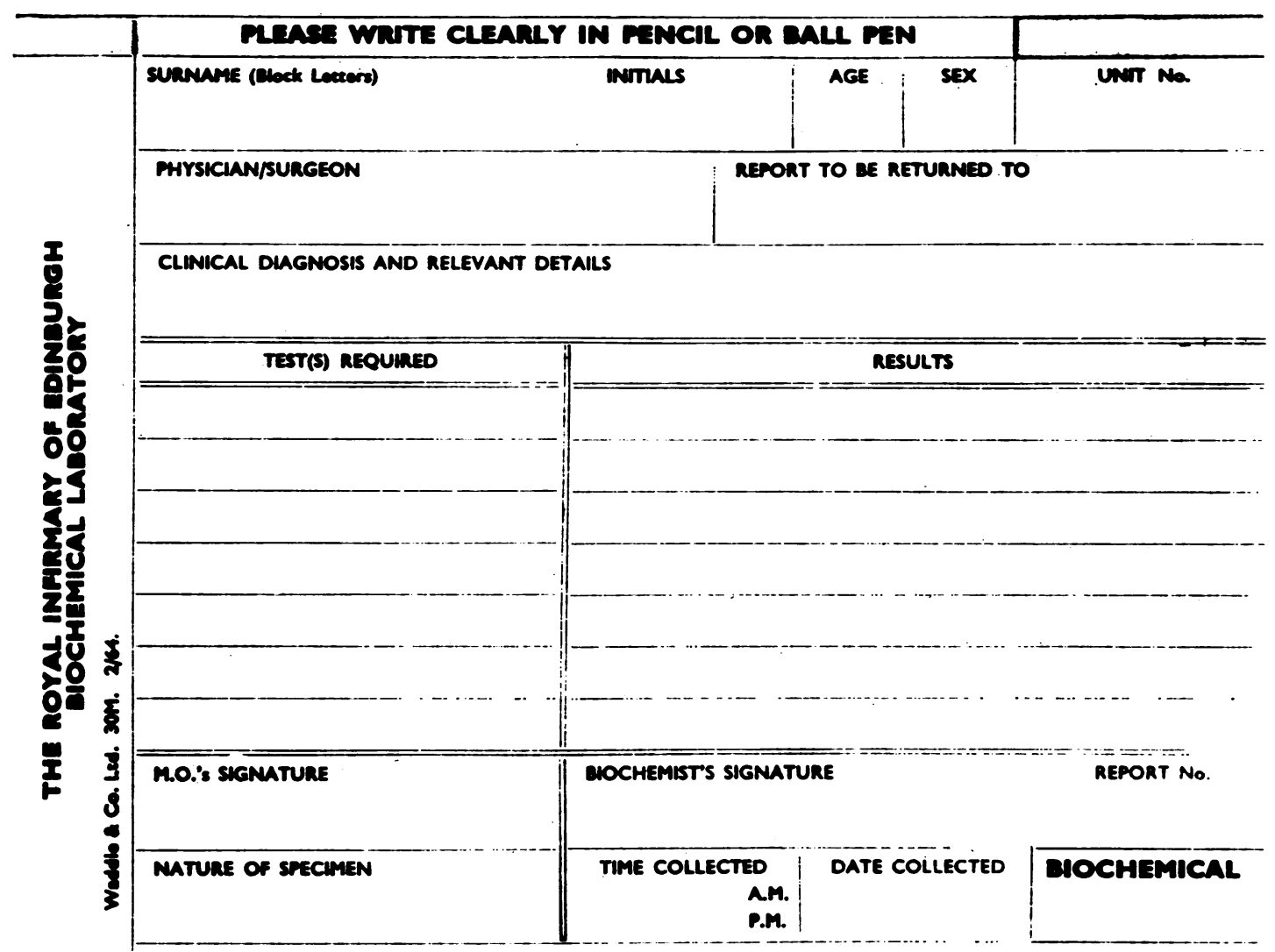

FIG. 1. Combined request and report form; a duplicate form is attached to the back and serves as the laboratory's record of work done.

THE ROYAL INFIRMARY OF EDINBURGH, DEPARTMENT OF CLINICAL CHEMISTRY

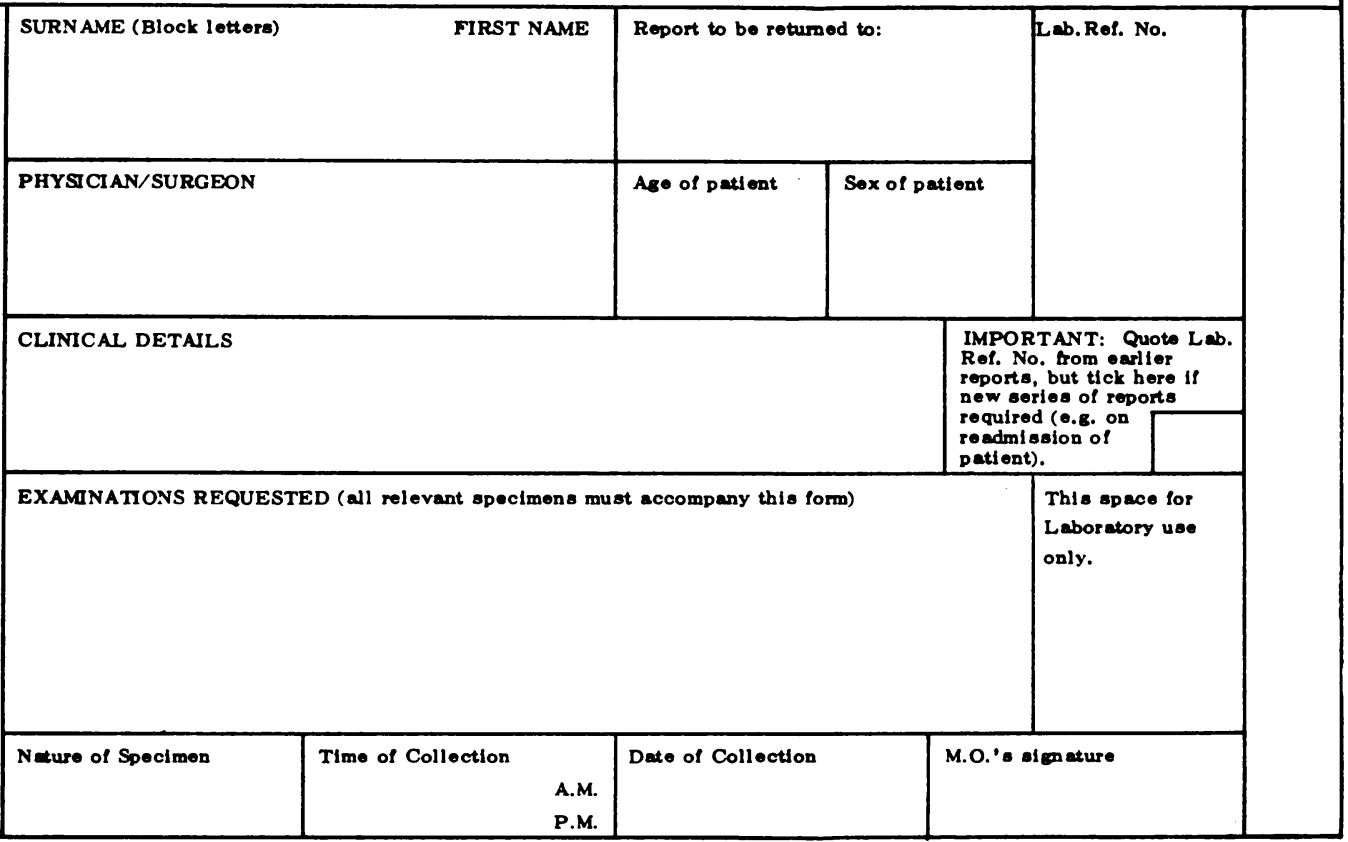

N.B. Please use black biro and prese firmly when completing request forms.

FIG. 2. Request form used by wards receiving serial and composite records of laboratory results. 
FLOW DLAGRAM FOR PROCESSING OF REQUESTS

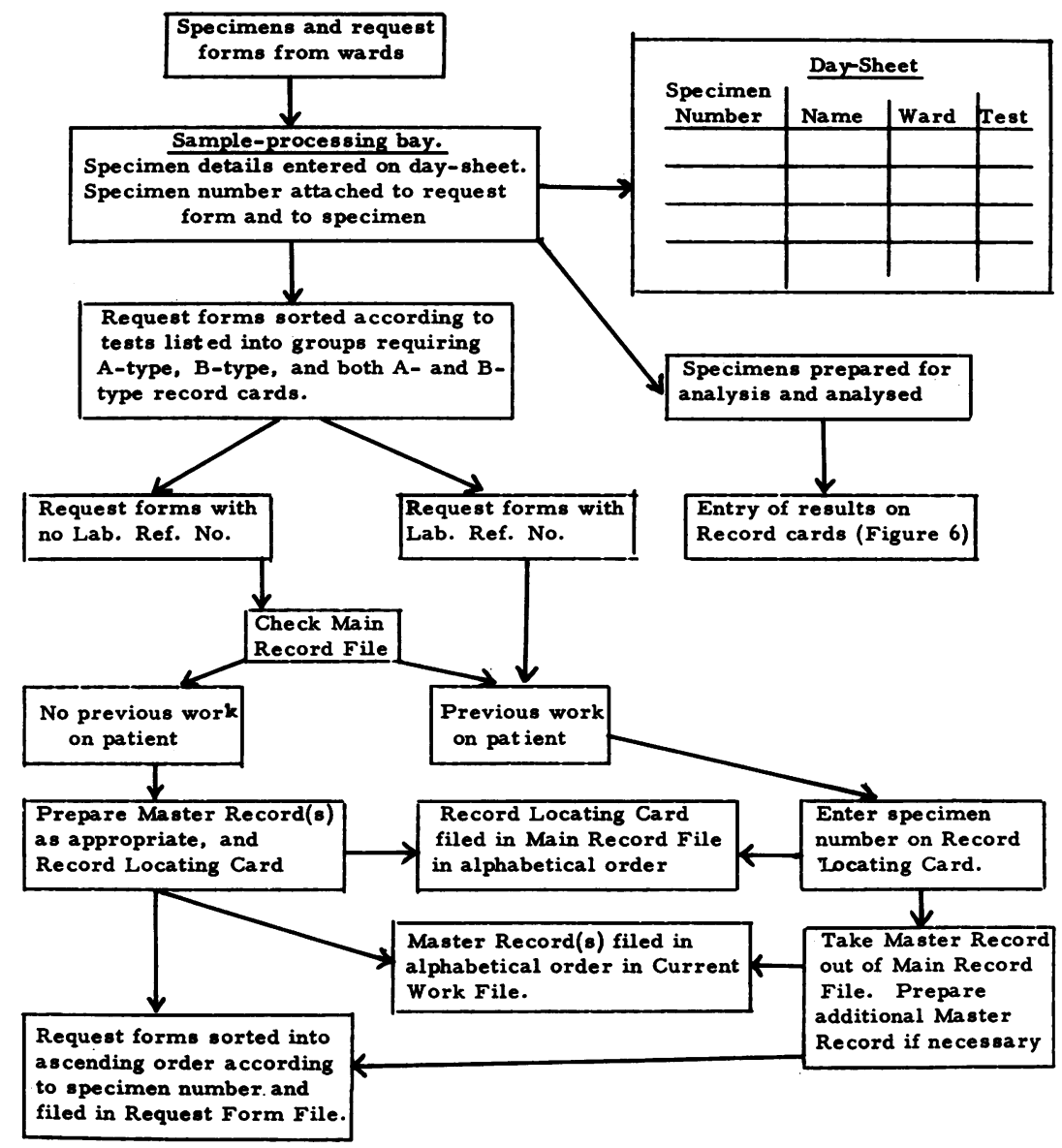

FIG. 3. Details of stages in the preparation of the main record file, current work file, and request form file.

required for each patient include a master record and a record locating card. Two types of preprinted master record are used (Figs. 4 and 5). The printing on the master records is restricted to an area of $10 \mathrm{in} . \times 7 \mathrm{in}$. but the cards themselves are wider (8 in.) to avoid edge-effects when copying.

When preparing master records, request forms are sorted into three categories: (a) tests limited to A-cards; (b) tests limited to B-cards; (c) tests requiring both $\mathrm{A}$ - and $\mathrm{B}$-cards for entry of results. These are then dealt with as follows:

1 Request forms are further divided into those bearing a laboratory reference number (lab. ref. no.) and those without. For this latter group, the main record file is examined to find whether work has previously been requested for these patients. The lab. ref. no. is a reference number assigned by the laboratory on a previous occasion for identification purposes, and signifies that the laboratory already holds a master record for that patient. It is used to provide positive identification of patients because the Royal Infirmary, Edinburgh, does not have a uniform system for identifying patients by unit number or registration number, but it is not otherwise an essential feature of the system.

2 For all first requests, a master record and a record locating card are prepared. The record locating card has a format for entry of patient details identical with the master records, but the lower part of the card consists of columns, headed A and $B$. The specimen numbers are entered under the appropriate headings (A, B or both) on the record locating cards, and cards and request forms are filed as follows: (a) record locating cards alphabetically in the main record file, where they remain at all times until a patient's cards are relegated to the file for old records; (b) master records alphabetically in the current work file; (c) request forms in the 


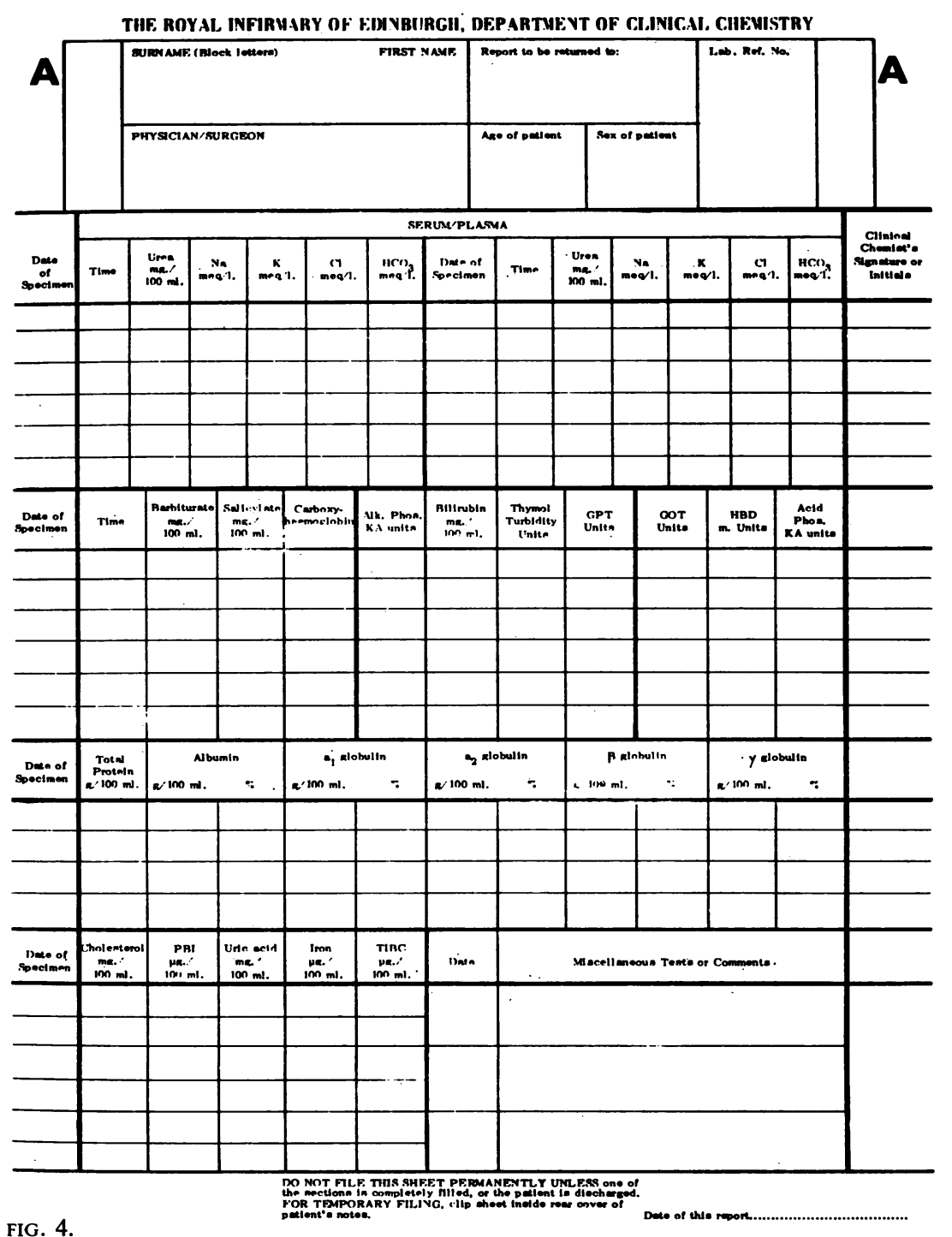

FIG. 4 and 5. Preprinted master record for entry of results. This card remains in the laboratory at all times. Copies of this card are issued, as reports, when results have been entered and the card signed in the corresponding right-hand column.

request form file in order of accession. The main record file and the current work file are held in Series 130 machine posting trays, style no. $133^{3}$.

3 For patients on whom work has previously been requested, the number of the specimen corresponding to the latest request is entered on the record locating card under the appropriate heading (A, B or both), and this card shows whether the requisite master record has already been made out.

If the latest request form requires a B-type master record, and the record locating card only has entries in the A-columns, a B-type master record is ${ }^{3}$ Roneo Ltd., 17 Southampton Row, London. prepared. The master records are then transferred to the current work file, For patients with previous work still outstanding, the master record will not be in the main record file. and the latest specimen number is entered on the record locating card. For patients having both types of master record and both A- and B-cards in the main record file when a new request is received, if the latest request is restricted to tests on the A-card, the A-card only is transferred to the current work file.

The current work file thus contains master records of patients for whom results of work have not yet been reported, and the request form file contains the 


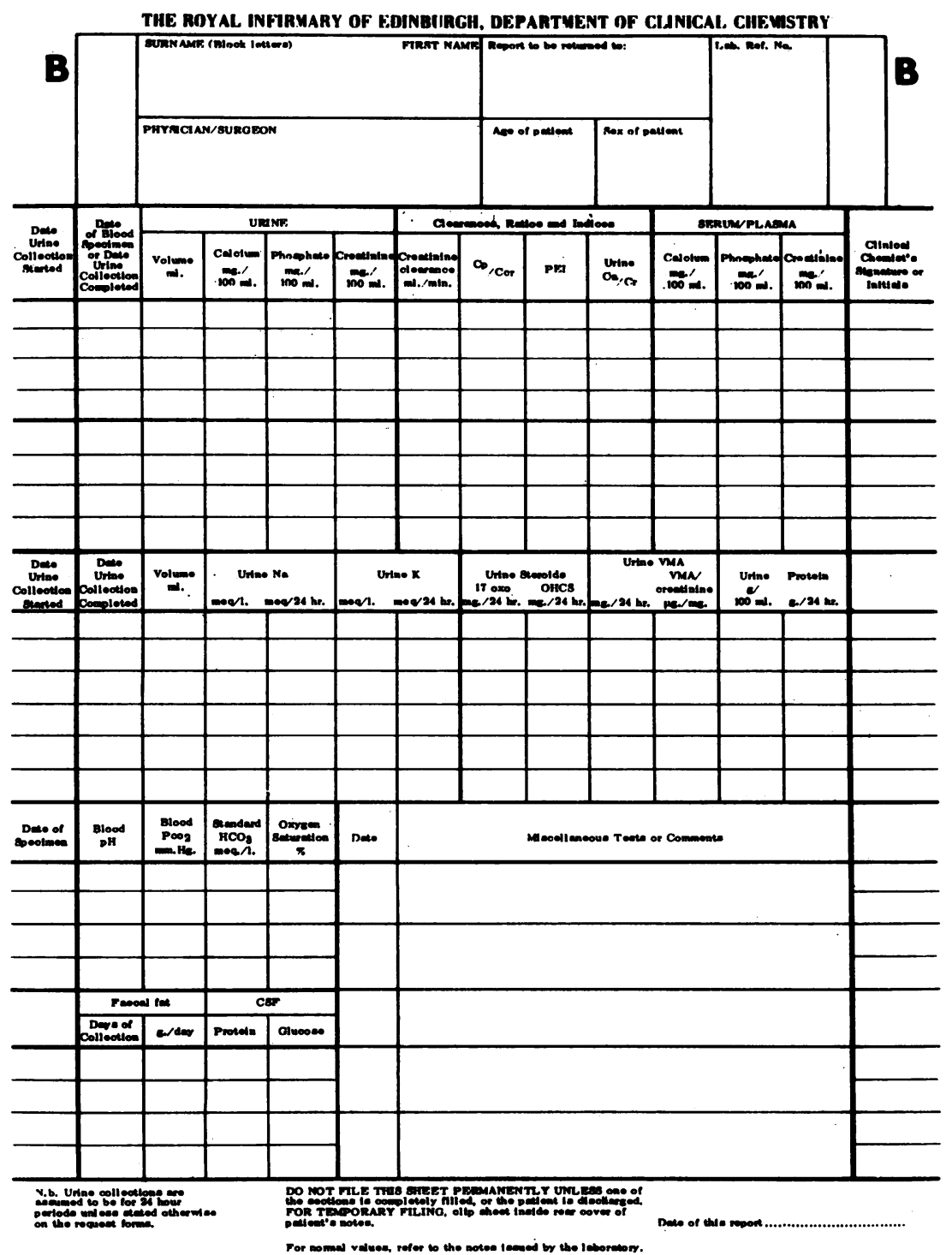

FIG. 5.

corresponding request forms. The main record file contains a record locating card for each patient who has had a master record prepared, and it contains the master records for those patients on whom all work has been completed and reported.

ENTRY OF RESULTS ON MASTER RECORDS (FIGURE 6) When results are ready for entry on master records, the request forms are taken out of the request form file by reference to each specimen's letter and number. The technicians now know the names of patients, and can find the master records in the current work file. Results are entered and request forms attached to their master records. These are then refiled in the current work file, unless the results are urgently required. This keeps these master records available as long as possible, before reporting, for the possible later entry of results by other technicians.

Results are entered with a black-ink ball-point pen. Any mistakes are covered with a self-adhesive ${ }^{4}$ label and the correct figure written on this. Comments in relation to analyses (e.g., specimen haemolysed; 'Tickotabs. Gosheron Ltd., 79-81 Albert Embankment, London. 


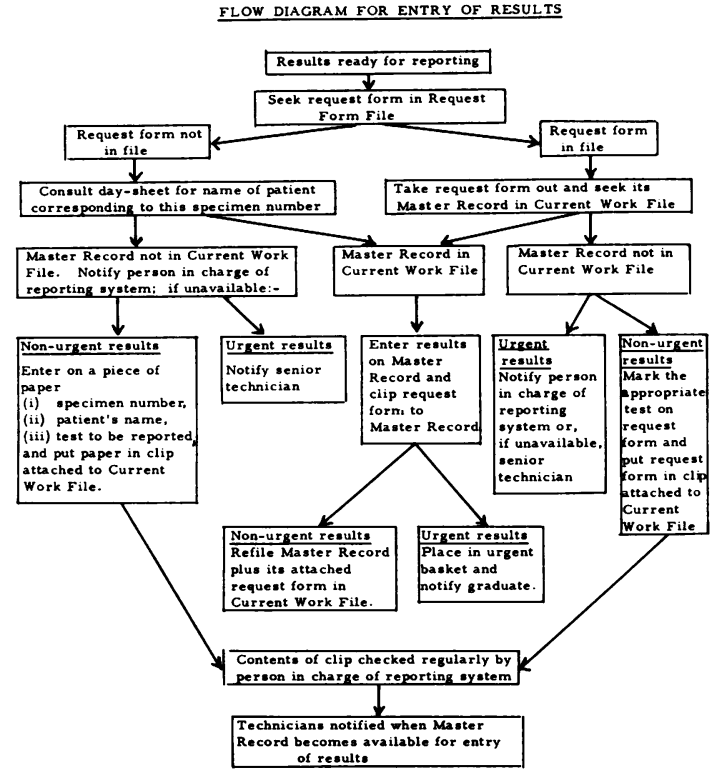

FIG. 6. Various steps performed by technical staff when entering the results of analyses on master records.

an abnormal band in the $\gamma$-globulin zone) are written by the technician on the request form, and the graduate, when signing the report, decides whether to enter these comments on the master records. No results are entered on a line which already bears a signature, except in the plasma electrolyte section, where a self-adhesive label is fixed over the column of signatures when the lefthand section has been filled.

Very occasionally technicians wishing to enter results cannot find the request form corresponding to the specimen number in their notebooks. There are two possible reasons for this:

1 When several different tests or groups of tests have been listed on one request form and more than one of these is ready for reporting at about the same time. When this occurs, the technician finds the patient's name by reference to the day-sheet and examines the current work file. If the request form and its master record are there, he enters the results, but otherwise he notifies the secretary responsible for the reporting system, who informs him later when the results can be entered. In the absence of the secretary, the technician puts details about the specimen on a slip of paper which is then left in a clip attached to the current work file.

2 With patients for whom more than one request form has work outstanding, and for whom results of tests on two or more request forms become ready for entry at about the same time. The technician may find the request form but then discover that the master record is not in the current work file, in which case he puts the request form in the clip attached to the current work file, making a mark on the request form against test(s) for which results are ready. The secretary goes through the contents of this clip regularly and notifies the technicians when the results can be entered.

If a technician has urgent work that cannot immediately be entered on the appropriate master record, the secretary locates the card specially for him; it will be found somewhere in the reporting part of the process.

PREPARATION OF REPORTS At least twice daily the current work file is examined and master records with request forms attached are taken out for inspection and signature by a member of the graduate staff, who checks the results which have been entered against the details on the attached request form. Very occasionally results have been found entered on another patient's card, but the most common mistake has been for today's date to be entered in the column headed date of specimen, although work may not have been completed on the day of collection. Master records with results requiring repetition of work before the issue of reports are set on one side until the rest have been inspected and passed for copying, as the reporting stage of the process must be kept short for the largest possible number of master records and request forms, if technicians are to find the maximum number of forms in the request form file, and of master records in the current work file, whenever they require them.

Any comments that will have permanent value are written by the graduate on the master record, but ephemeral comments are entered on the report. When signing, the graduate crosses the corresponding test(s) off the request form, and divides the master records into two piles, ready for copying, keeping the master records interleaved with their corresponding request forms. The two piles are: (i) master records with completed requests; (ii) master records with request forms that still have tests outstanding. This latter pile has priority in the next stage of the reporting process, and its request forms and master records are then returned to the request form and current work files.

Copying master records A Rank-Xerox 914 office copier is used for preparing reports on $10 \mathrm{in}$. $\times 8$ in. plain pink paper, perforated with two holes on the left-hand margin. A template is fixed on the scanning glass so that the master record can be rapidly aligned in relation to the paper that is to receive the copy. The date on which copies are being made is typed on a small piece of paper and fixed to 
the scanning glass with Sellotape so as to overlie the space next to 'date of this report ...', at the lower right-hand corner of the master records (Figs. 4 and 5). Throughout the process of preparing reports, master records and their request forms are kept together, to assist in subsequent refiling, and in case any unexpected checks of individual cards or forms should prove necessary at any time.

FILING REPORTS IN THE PATIENTS' RECORD FOLDERS Reports are sorted according to wards, placed unfolded in plastic open envelopes, and issued. The envelopes are returned later for reuse. Instructions about the filing of reports are printed at the bottom of the master record (Figs. 4 and 5) and appear on each copy report. Each time a new report is issued, it replaces the previous report in the patient's folder; the information on this earlier report is reproduced in full on the later report, together with the results of the latest series of tests. It is necessary to check whether the latest report is a copy of the A-type (Fig. 4) or B-type (Fig. 5) of master record before discarding a previous report.

REFILING MASTER RECORDS WHEN ALL TESTS ON THE ACCOMPANYING REQUEST FORMS HAVE BEEN REPORTED These master records are matched against their record locating cards in the main record file, and the specimen numbers on the request forms crossed off the record locating cards. If there is no longer a specimen number remaining under the heading (A or B) on the record locating card corresponding to the master record, the latter is refiled in the main record file. If, however, another specimen number still remains under this heading, there is another request form with work still outstanding for the patient and the master record is accordingly refiled in the current work file.

MISCELLANEOUS POINTS Specimens of heparinized blood, clotted blood, urine, etc., from one patient each receive a different consecutive identification number even though they relate to the same request form, to facilitate identification of specimens. Blood sugar analyses are reported separately on forms designed for serial presentation of results in patients' notes (Fig. 7).

\section{PRACTICAL EXPERIENCE WITH THE SYSTEM}

The system has been used for seven months. For the first four weeks it was limited in application to patients occupying 140 beds, and was then extended to cover a further 110 beds. The results reported here are based on the master records of all patients admitted to these beds during the period 27 July

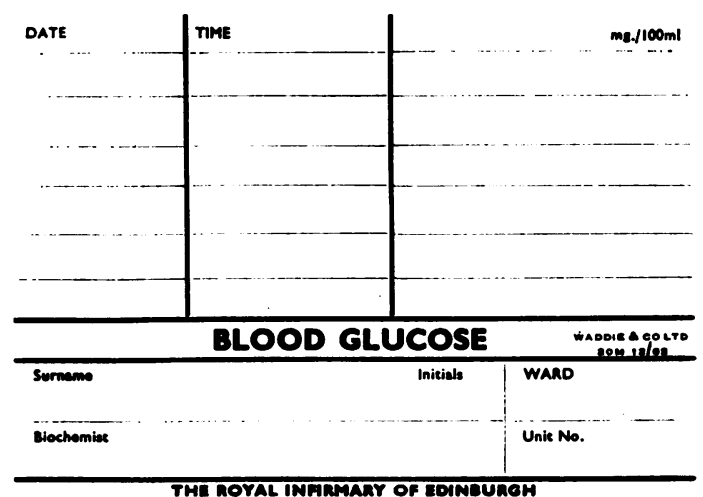

FIG. 7. Form used for results of blood sugar determinations. By overlaying reports, details about the patient on $\infty$ the preceding report are covered and a serial record is 응 obtained.

1964 to 28 February 1965. Patients who were already in the wards when the method of reporting was changed have been omitted if they were discharged in the next 12 days, and if they had only had a single investigation or group of tests (v. infra) performed on one occasion in this period (48 patients); no other patients have been excluded. Patients admitted after 16 February 1965 have been followed up to 12 March before completing the analysis of their record cards. The average duration of stay for patients in the Royal Infirmary, Edinburgh, is 12 days (Scottish Home and Health Department, 1965).

Table I shows the number of patients for whom master records were prepared, and details of the requirements for each type of card. Table II sum-

\section{TABLE I}

NUMBER OF PATIENTS FOR WHOM MASTER RECORDS HAVE BEEN PREPARED

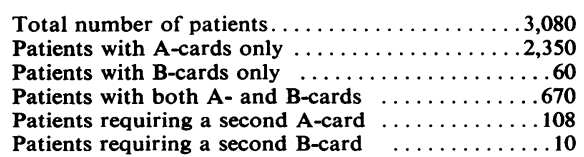

marizes the use that has been made of these cards. $N$ Various terms introduced in Table II require $\underset{\omega}{ }$ definition:

'A group of tests' refers to a set of analyses all of 0 which are usually performed in this laboratory $\mathbb{D}$ whenever one or more of the group is requested, ? e.g., plasma urea and electrolytes, liver function tests, protein electrophoresis. A single set of results for one of these groups has been counted as a single entry. 
TABLE II

DETAILS OF ENTRIES MADE ON MASTER RECORDS

Number of Patients Proportion (\%) of All Patients for whom Either (A-or B-Master Records Prepared ${ }^{1}$

Entries under one test or group of tests only

(a) Single entries

Entries on A-cards

Entries on B-cards

(b) More than one entry, i.e., serial records

Entries on A-cards

Entries on B-cards

Entries under more than one test or group of tests on one type of master record (A-or B-card)

(a) Single entries under more than one test or group of tests, i.e., composite records

Entries on A-cards

Entries on B-cards

(b) More than one entry under one or more test or group of tests, i.e., both serial and

composite records

Entries on A-cards

Entries on B-cards

$\begin{array}{rr}887 & 29 \cdot 4 \\ 336 & 46 \cdot 0 \\ 346 & 11 \cdot 5 \\ 75 & 10 \cdot 3\end{array}$

${ }^{1}$ The total number of patients with A-cards is 3,020 and with B-cards is 730 .

'A single entry' is an entry of the result for one test or group of tests. Single entries never occupy more than one horizontal line.

'A serial record' includes two or more results, for the same test or group of tests, obtained on two or more occasions. Serial records allow comparison of the latest set of analytical results with previous findings for the same test(s).

'A composite record' includes on one master record results of analyses under two or more apparently unrelated headings.

Table II shows that $70.6 \%$ of all A-cards prepared and $54.0 \%$ of all B-cards contained either composite records or serial records or both types of information.

On its present scale (approximately 450 reports per week), the system requires the services of a secretary for about two-thirds of her time. She collects the request forms after entries have been made on the day-sheet, and is thereafter in charge of all stages of the process except for the inspection and signing of master records. One graduate spends approximately one to one and a half hours daily examining and signing master records, and a varying amount of extra time investigating results which require checking before issue. The technical staff enter the results on the master records; this does not take them appreciably longer than entering results on the forms (Fig. 1) used for the rest of the laboratory work.

Running expenses are detailed in Table III, in terms of three different examples. Most of the use

TABLE III

EXAMPLES OF COST OF REPORTING SYSTEM

\begin{tabular}{|c|c|c|c|}
\hline & & Cost (Penni & \\
\hline & Example 1 & Example 2 & Example 3 \\
\hline Request form(s) & $0 \cdot 15$ & $1 \cdot 80$ & $0 \cdot 30$ \\
\hline Master record(s) & 0.94 & $1 \cdot 88$ & 0.94 \\
\hline Record locating card & $1 \cdot 32$ & $1 \cdot 32$ & $1 \cdot 32$ \\
\hline Report paper & 0.32 & 3.94 & $3 \cdot 20$ \\
\hline Rank-Xerox unit copy charge(s) & 3.00 & 36.00 & 30.00 \\
\hline Proportion of costs of: & & & \\
\hline Drum replacement ${ }^{2}$ & 0.35 & $4 \cdot 20$ & $3 \cdot 50$ \\
\hline Consumable items needed for Rank-Xerox 914 (i.e., developer, toner, & & & \\
\hline $\begin{array}{l}\text { filter bags, rayon wool, film remover) } \\
\text { Total cost }\end{array}$ & $\begin{array}{l}0 \cdot 7 \\
7 \cdot 38\end{array}$ & $\begin{array}{c}8 \cdot 4 \\
64 \cdot 74\end{array}$ & $\begin{array}{c}7 \cdot 0 \\
52 \cdot 26\end{array}$ \\
\hline Cost per report & $7 \cdot 4$ & 5.4 & $5 \cdot 2$ \\
\hline
\end{tabular}

Example 1 A single request requiring one master record and one report.

Example 2 Twelve single requests, some requiring an A- and others a B-type master record, and generating reports that were issued on 12 different occasions.

Example 3 Two multiple requests, needing an A-type master record only and generating reports that were issued on 10 different occasions.

${ }^{1}$ On basis of 2,000 copies per month; this figure falls with greater usage of the machine.

${ }^{2}$ Replacements cost $£ 29$; estimated life, 20,000 copies. 
has fallen between these limits, and the average cost per report has been 6.0d., including an allowance for the occasional spoiled copy and for the few instances where patients have been issued with more than one lab. ref. no. To these running costs should be added part of the secretary's salary. Capital costs included the machine posting trays ( $£ 8$ each), and the installation costs of the Rank-Xerox 914 machine.

\section{DISCUSSION}

This system of reporting chemical analyses has several advantages for the clinician. Request forms can be used for multiple requests and, unless a large number of tests have to be requested, the reports of chemical investigations (except for blood sugar results) appear in the notes on two sheets of paper. Results of serial investigations can be viewed at a glance and transcribing of chemical results from individual reports for the preparation of serial records on the wards has been eliminated. These reports have made it easier to follow chemical changes occurring during the course of a patient's illness, and composite laboratory reports have probably contributed therefore to the welfare of patients.

The system of collecting results on to master records has had many advantages in the laboratory. With serial results particularly, these records have helped to control laboratory accuracy by revealing occasional grossly discordant and erroneous results, and have added to the interest of work by allowing staff to observe the progress of laboratory findings on patients, thereby also helping to maintain accuracy. Access to departmental records has been simplified, since a complete chemical history for the patient can be consulted easily. Master records on patients have not been employed to limit the use made of laboratory facilities, although they might reveal possible instances of over-repetitious requests.

The main disadvantage is cost, but the expense of serial reports should be related to the total unit cost of chemical investigations, which was 11s. per request in 1964, calculated on the basis adopted in Hospital O. and M. Service Report 6 (1963). To extend the system of reporting described here to cover the whole Royal Infirmary will require three extra secretaries, and the total increase in unit cost resulting from wages and operating costs directly connected with the system will be 8-9d. per request. This assumes that each request continues to be equivalent, on average, to three chemical tests, which was the pattern in 1964 .

The above comparison of costs is based on the continued use of the present request form (Fig. 2), but patient details (including registration number) are shortly to be incorporated into an Addressograph $^{5}$ stamp and each first request for chemical investigations will be accompanied by two spare Addressograph labels. Redesigning the top of the master records will accommodate these labels, shorten the time taken preparing master records and is record locating cards, render the lab. ref. no. $\vec{\circ}$ unnecessary, and reduce by one the number of secretaries needed to run the system for the whole Royal Infirmary, thereby saving approximately $1 \mathrm{~d}$. per request. A further saving of 1d. per request will occur when the Rank-Xerox 914 is replaced by a $\vec{\infty}$ Bruning 2000, which operates about twice as fast $i r$ and at lower cost. With these modifications, the increase in unit cost will become about $6 \mathrm{~d}$. per report.

In the system described by Mason (1962), an individual record card is prepared for each patient in the laboratory, the layout depending on the nature of investigations requested. Descriptive headings of tests, printed on adhesive labels, are fixed as required at the head of columns on record cards. Lack of standardization in the position of column headings must prevent laboratory staff from looking automatically at the same place on each patient's master record, when entering the results of a particular test or group of tests, and clinicians must be at a similar disadvantage when examining reports. Preprinted master records do not suffer from this disadvantage.

This paper describes practical points which become important when the serial records system is introduced into a laboratory with a large technical staff, all of whom require access to master records. The current work file keeps the technicians away from the bulky main record file, and the record locating card controls the movement of each patient's master records. It should be mentioned that, initially, master records printed on both sides and incorporating on one card the headings now provided on two cards (Figs. 4 and 5) were used. Only $21 \%$ of the first 700 patients had entries on both sides and, with these cards, it was not always obvious which side of the card carried results ready to be reported, so master records printed on only one side were preferred.

When this system is extended to cover all patients served by this laboratory, separate arrangements will 6 be needed for general practitioners, because $10 \mathrm{in}$. $\times 8$ in. reports are too large to be kept in patients' medical record envelopes, and the method in operation at the General Hospital, Margate (RankXerox Ltd., 1963), will be closely followed.

${ }^{5}$ Addressograph Multigraph Ltd., Hemel Hempstead, Herts. 
If the work of this department continues to rise exponentially, as in previous years (Whitby, 1963), electronic data-processing equipment will become essential. However, such equipment will probably be too expensive for all but the largest hospital groups for several years, if it is to provide serial and composite reports and laboratory records as quickly as the manual system described here. The present system has been described in detail, therefore, because it can provide, at reasonable cost, reports and records that incorporate most of the features which can be gained from the use of much more expensive computer-dependent reporting techniques.
The authors wish to acknowledge the help received from Mr. G. K. Leslie of Rank-Xerox Ltd. during the establishment of this reporting system, and to thank members of the departmental staff, particularly Miss E. E. Williams, for comments and suggestions upon its operation.

\section{REFERENCES}

Ministry of Health. (1963). Pathology-Measurement of Work in Units (Hospital O and M Service Report no. 6), p. 55. H.M.S.O. London.

Mason, W. B. (1962). Ann. N.Y. Acad. Sci., 102, 171.

Rank-Xerox Ltd. (1963). Leaflet $R X 131$.

Scottish Home and Health Department (1965). Analysis of Running Costs of Scottish Hospitals, p. 8. H.M.S.O., Edinburgh.

Whitby, L. G. (1963). Lancet, 2, 1239. 